\title{
ComEst: a Completeness Estimator of Source Extraction on Astronomical Imaging
}

\author{
I-Non Chiu ${ }^{\mathrm{a}, \mathrm{b}}$, Shantanu Desai ${ }^{\mathrm{b}}$, Jiayi Liu ${ }^{\mathrm{c}}$ \\ ${ }^{a}$ Faculty of Physics, Ludwig-Maximilians University, Scheinerstr. 1, D-81679 München, Germany \\ ${ }^{b}$ Excellence Cluster Universe, Boltzmannstr. 2, 85748 Garching, Germany \\ ${ }^{c}$ Bosch Research and Technology Center North America, 4005 Miranda Ave \#200, Palo Alto, CA 94304, United States
}

\begin{abstract}
The completeness of source detection is critical for analyzing the photometric and spatial properties of the population of interest observed by astronomical imaging. We present a software package ComEst, which calculates the completeness of source detection on charge-coupled device (CCD) images of astronomical observations, especially for the optical and near-infrared (NIR) imaging of galaxies and point sources. The completeness estimator ComEst is designed for the source finder SExtractor used on the CCD images saved in the Flexible Image Transport System (FITS) format. Specifically, ComEst estimates the completeness of the source detection by deriving the detection rate of synthetic point sources and galaxies simulated on the observed CCD images. In order to capture any observational artifacts or noise properties while deriving the completeness, ComEst directly carries out the detection of simulated sources on the observed images. Given an observed CCD image saved in FITS format, ComEst derives the completeness of the source detection from end to end as a function of source flux (or magnitude) and CCD position. In addition, ComEst can also estimate the purity of the source detection by comparing the catalog of the detected sources to the input catalogs of the simulated sources. We run ComEst on the images from Blanco Cosmology Survey (BCS) and compare the derived completeness as a function of magnitude to the limiting magnitudes derived by using the Signal-to-Noise ratio (SNR) and number count histogram of the detected sources. ComEst is released as a Python package with an easy-to-use syntax and is publicly available at https://github.com/inonchiu/ComEst .
\end{abstract}

Keywords: survey: photometry: depth: completeness:

\section{Introduction}

The optical and Near-Infrared (NIR) imaging provides one of the backbones of astronomy. Since the early twentieth century, countless milestones have been made in various fields of astronomy based upon the data taken by the optical sky surveys, e.g., SDSS (York et al., 2000). Nowadays wide field optical/NIR surveys- such as the Pan-Starrs (Morgan et al., 2014), DES (Flaugher. 2005), KiDS (de Jong et al., 2013), HSC (Miyazaki et al., 2012), 2MASS (Skrutskie et al., 2006), WISE (Wright et al., 2010) and ATLAS (Shanks et al. 2015)- and dedicated deep imaging (e.g., COSMOS (Koekemoer et al. 2007) or HUDF (Beckwith et al. 2006) ) have become the frontier of astronomical studies in various topics. In the next decade, the upcoming surveys- for instance, LSST (Ivezic et al. 2008) or Euclid (Laureijs et al., 2011) - with the unprecedented deep imaging of large portion of the sky will revolutionize our understanding of the Universe.

Nowadays, modern astronomical observations are imaged by charge-coupled devices (CCD) saved in the Flexible Image Transport System (FITS) format, which various source finders, e.g., (Bertin and Arnouts, 1996) are run on for detecting sources. To analyze the observed images taken by various telescopes, the astronomical objects are identified by the source finder, and then information about the photometric and spatial properties is extracted from the image. It is therefore critical to verify the source detection on the observed image for the analysis of, for instance, modeling the luminosity function or spatial clustering of galaxies. One of the most important factors for such studies is to quantify the completeness of the source detection on the observed images.

The completeness of the source detection indicates the fraction of objects present in the image, which can be detected by the source finder above a certain detection threshold against the noise. The completeness is expected to decrease with increasing detection threshold because the required signal-to-noise ratio (SNR) of the source is higher. At the same time, the purity of the source detection- the fraction of the detected sources which are not spuriously detected by the source finder- becomes higher due to the high SNR as well. The lack of understanding the completeness (or purity) of the source detection could lead to biased scientific results. For example, the modeling of luminosity function at the faint end would be biased if one does not pay special care to the completeness near the detection threshold.

There are several ways to quantify the completeness of the source detection in terms of limiting depth (or limiting magnitude). In general, one can compare the source catalog, which is extracted from the observed image by the source finder, to a reference catalog built from the image with deeper depth. By comparing the source counts normalized by the observed area between two catalogs, one can calculate the completeness as the ratio of source counts given a limiting magnitude. For example, the source count-magnitude relation assuming a power law, with the power law index estimated from the reference catalog can be fitted to the source catalog to determine the completeness, which is defined as the ratio of the number counts of the source catalog to the best-fit power law model at given magnitude (e.g., Zenteno et al. 2011: Chiu et al., 2016). However, this approach ignores

Email addresses: inonchiu@usm.lmu.de (I-Non Chiu), shantanu@usm.lmu.de (Shantanu Desai), jiayiliu@usm.lmu.de (Jiayi Liu) 


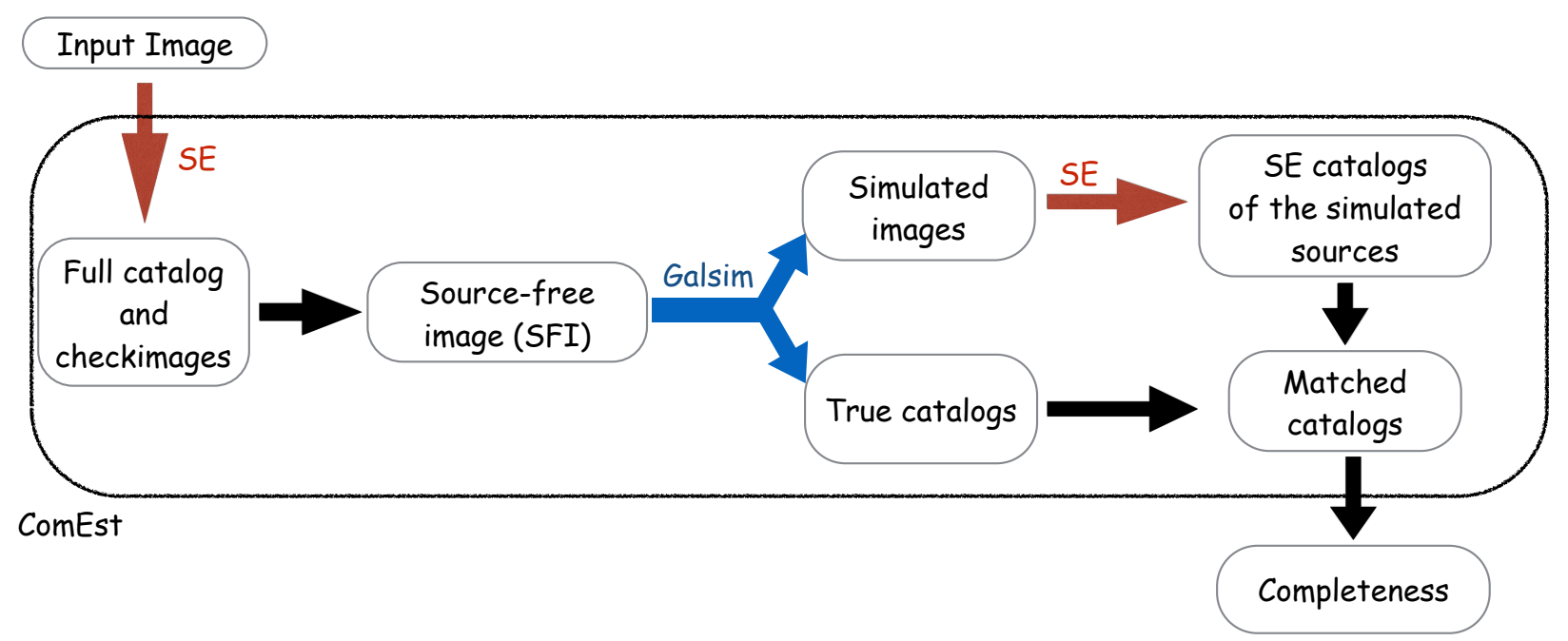

Figure 1: The workflow of ComEst. The input image in the FITS format is the only input required by ComEst. Once the input image is loaded, ComEst runs SExtractor to return the check-images and use the check-images to create the source-free image (SFI). Then ComEst simulates various synthetic sources on SFI where SExtractor is run on to detect the simulated sources. Finally, ComEst matches the true catalog and the SExtractor catalog to derive the completeness.

the cosmic variance of the source properties on the sky and is frequently affected by the systematics between the catalogs, such as the difference in the observed filter systems, detection algorithms or the observational conditions. Another example is to link the photometric uncertainties of the detected sources to the completeness as a function of the survey depth (Rykoff et al. 2015). Nevertheless, this method requires intensive modeling of the sky noise and photometry measurement; in addition, the large number of extracted sources in the overlapping region between the source and reference catalogs is needed in order to obtain the precise completeness estimates.

On the other hand, the most direct way to estimate the completeness of the source detection is to quantify the performance of the source finder by running the same detection algorithm on the objects simulated on the observed image. In this way, the completeness (and purity) of the source detection can be derived by comparing the extracted catalog to the catalog of simulated sources used as the input of the simulation. Simulating the sources on the observed image preserves any observational artifacts and sky noise while quantifying the performance of the source detection. Moreover, carrying out exactly the same algorithm to detect the simulated sources on the observed image provides an end-to-end verification and prevents any systematics arising from the catalog comparison. Several packages exist which can estimate the completeness using a similar methodology mentioned above, for example DAOPHOT (Stetson, 1987) or 2DPHOT (La Barbera et al., 2008). However, DAOPHOT is specifically designed for stellar-like objects and is not optimized for the extended sources such as galaxies. For the latter example, 2DPHOT requires intensive pre-modeling of the source properties (e.g., the morphology). In addition, the packages mentioned above are less user-friendly compared to the other image simulation software, such as GalSim (Rowe et al., 2015). A more straightforward way for source simulation is to simulate the sources based on the models with various properties, so that we have full control and are independent from the observed sources.

In this paper, we present the user-friendly software package ComEst-which is designed for estimating the completeness of the source detection on the observed image saved in the FITS format, by deriving the detection rate of the sources simulated with various properties assigned by the users. In addition, ComEst is designed for the source finder SExtractor (Bertin and Arnouts, 1996), which is widely used in the astronomy community for detecting the sources observed by the optical/NIR imaging. This paper is organized as follows. The methodology of ComEst is described in Section 2, while we demonstrate the usage of ComEst with an example and present the results in Section 3 . We conclude made in Section 4

\section{Methods}

ComEst is developed as a completeness estimator for the SExtractor cataloging program for any given imaging of point sources or galaxies with well-calibrated photometric zeropoint (ZP). To capture any observational effects which are already present in the image, ComEst directly simulates sources with various properties (e.g., the sizes or fluxes) and runs SExtractor to carry out the source detection on the observed image. Therefore, by design, ComEst heavily relies on the source finder SExtractor and the image simulation toolkit GalSim (Rowe et al., 2015), which is used as the engine for simulating sources.

The workflow of ComEst is described as follows. For a given CCD image, ComEst first runs SExtractor to detect the observed sources and returns a set of standard outputs, especially the check-images. Then, ComEst removes the detected sources from the observed image and replaces them- in the same positions- by the background values estimated by SExtractor. In this way, ComEst creates the source-free image (SFI) with the observed noise properties only. Next, ComEst puts various sources simulated by GalSim (Rowe et al., 2015) on the SFI to create a set of simulated images. After creating a set of simulated images, ComEst re-runs SExtractor again to detect the simulated sources. Finally, ComEst derives the completeness as a function of source flux and image position, by comparing the SExtractor catalogs of the simulated sources and the true catalog used as the input to the simulation. The workflow is shown in Figure 1 . We describe the details of ComEst in this section. 


\subsection{Source detection and the source-free image (SFI)}

The Source Extractor SExtractor (Bertin and Arnouts, 1996) is used as the source finder in ComEst. SExtractor is a program which detects the sources on the images and performs the photometric measurements of them. Since the first version in 1996, SExtractor has been recognized as the standard tool for detecting sources and estimating the photometry on the images in modern astronomy. For a given image and the SExtractor configuration, SExtractor builds the catalog of the sources detected on the images and returns a set of diagnostic outputs, such as the check-images. It is worth mentioning that the performance of the SExtractor depends on the configuration file provided by the user. We refer readers to the SExtractor manual 1 or Holwerda (2005) for more details about the optimized usage of SExtractor.

We stress that the observed image saved in the FITS format is the only input required by ComEst. The input image must contain the World Coordinate System (WCS) information in the header, which ComEst extracts to get information about the astrometry. ComEst first runs SExtractor on the input image to detect all the sources with fluxes above the threshold assigned in the configuration file, then SExtractor returns the output catalog and a set of check-images. The goal of the first run of SExtractor is to identify all the observed sources and estimate the background values on the image. Once the sources are identified, ComEst replaces the values in the pixels where the source is located by randomly sampling the background values assuming the normal distribution with the mean and standard deviation in the check-images with the types of BACKGROUND and BACKGROUND_RMS, respectively, estimated by SExtractor. In this way, ComEst creates the source-free image (SFI) with only the observed noise properties present. It is worth mentioning that the SFI image is- by design- free from the sources defined by SExtractor configuration (e.g., DETECT_THRESH or PHOT_AUTOPARAMS), therefore the sources undetected by SExtractor (e.g., below the detection threshold) and the fluxes outside the extracted apertures remain the properties of the observed background on the SFI, resulting in negligible impact on the detections of other sources which are in the higher signal-to-noise regime (also see Section 2.3). Running SExtractor with the weight-map and other additional arguments is supported in ComEst.

Alternatively, ComEst can also remove only the sources which satisfy a certain criteria from the image and keep the others at the same positions. Specifically, ComEst can only remove the detected sources for which the fluxes (and sizes) are fainter (and smaller) than certain thresholds assigned by the SExtractor arguments (DETECT_THRESHOLD and DETECT_MINAREA) and keep other observed sources on the image. This is particularly useful when one wants to estimate the impact on completeness from the area masked by the relatively brighter and bigger sources on the image (Umetsu et al. 2011).

\subsection{Simulating sources}

ComEst generates the simulated images by placing the simulated sources on the SFI, which only keeps the artifacts and noise properties observed in the image. The python module of the simulation toolkit-GalSim- is used as the central engine for simulating sources in ComEst. Two kinds of simulated sources are supported in ComEst; the first one is the point sources (stellar-like sources) and the second one is the galaxies. While simulating the sources (point source or galaxy), each source is randomly placed on the SFI with the magnitude (or the flux) uniformly distributed in the interval assigned by the user. It is important that the well calibrated ZP of the input image must be provided to ComEst for simulating the sources with unbiased photometry.

For simulating the point sources, ComEst simply convolves each source with the Point Spread Function (PSF) using the assigned Full Width at Half Maximum (FWHM). On the other hand, ComEst simulates galaxies- convolved with the PSFwith the user-defined inputs for the half-light radius, major-to-minor axis ratio, and the position angle. In addition, ComEst currently provides two kinds of schemes for simulating galaxies: one can construct each galaxy by mixing the bulge and disk components with certain fractions, or one can also directly simulate the galaxies re-sampled from the galaxy catalog observed by the COSMOS survey (Ilbert et al. 2009; Rowe et al., 2015).

ComEst simulates the sources on the SFI according to the pre-determined projected number density on the sky ${ }^{2}$. The number density of the simulated sources is assigned by users to avoid the blending problem in crowded fields. In this way, ComEst can avoid the incompleteness of the source detection, which is actually due to blending in the crowded field instead of the sky noise observed in the image. To achieve better statistics in deriving the completeness, ComEst can generate multiple simulated images with the same simulation scheme described above. Simulating multiple images can be expedited by running GalSim using multiple threads, which is currently supported in ComEst. In the end, ComEst returns the simulated images and the true catalogs which contain the input parameters for simulating the sources.

\subsection{Deriving completeness and purity}

After simulating the sources on the SFI, ComEst re-runs SExtractor again on the simulated images to generate the SExtractor output catalogs. It is important that the same configuration, which is used in the first run of SExtractor in ComEst (see Section 2.1), is also used for detecting the simulated sources, in order to have the derived estimates (e.g., the completeness of the detection) which are estimated from the identical process of the source extraction.

It is important to note that ComEst assumes that the undetected sources- which are present on the observed image but are not detected in the first run of SExtractor- remains undetected on the SFI with the same SExtractor configuration andadditionally- they have negligible impact on the detectability and photometric measurement of the simulated sources higher than

\footnotetext{
${ }^{1}$ http://www.astromatic.net/software/sextractor

${ }^{2}$ In addition to simulating galaxies with the pre-determined projected number density, ComEst also provides an utility (comest. AnalysisSEcat) to produce the simulated galaxy catalogs based on the magnitude distribution in the SExtractor catalog extracted from the observed image. In this way the source detection affected by the blending can be addressed.
} 
the detection threshold. It is a reasonable assumption for the majority of cases where the source detections are not dominated by the background, i.e., the signal-to-noise of the source is well above the detection threshold. On the other hand, the undetected sources near the confusion limit (e.g., the signal-to-noise ratio near to the DETECT_THRESH in SExtractor) contribute additional noise to the source detection on the SFI, hence the completeness estimates are likely to be biased low near the detection threshold, as a caveat of ComEst. Therefore, the users should pay attention to the SExtractor configuration based on the magnitude range of interest where the completeness or purity properties are estimated.

After the source detection on the SFI, ComEst matches the simulated sources detected by SExtractor to the sources in the input catalog. Specifically, for each detected source ComEst first looks for the counterparts in the input catalog within the positional tolerance set by the users (default value is one FWHM), followed by the procedure of magnitude filtering such that the measured and input magnitudes are within the magnitude threshold (default is $1.5 \mathrm{mag}$ ). That is, a matched pair has to be within the positional and magnitude tolerance. If there are multiple objects within the positional tolerance in the first phase of matching, then the one within the magnitude threshold and with the closest magnitude difference is considered to be the matched object. After visually inspecting the matched pairs, we find that majority of the matched pairs do not have multiple counterparts in the position matching for the number densities seen on the realistic sky, and adding the magnitude filtering after the positional matching can effectively remove the spurious matched pairs for the extreme case of $\approx 200$ galaxies/arcmin ${ }^{2}$.

If the multiple simulated images are produced upstream, ComEst will automatically merge the SExtractor catalogs and the true catalogs of all the simulated images.

Once the output catalogs of the simulated sources are built, ComEst estimates the completeness as a function of magnitude (Equation 1) by comparing the fraction of the input sources recovered by SExtractor.

$$
\mathrm{f}_{\text {com }}(m)=\frac{N_{\text {se }}(m)}{N_{\text {true }}(m)},
$$

where $\mathrm{f}_{\text {com }}(m), N_{\text {se }}(m)$ and $N_{\text {true }}(m)$ are the completeness, number of simulated sources re-covered by SExtractor and the number of input sources in the simulation, respectively, at a given magnitude $m$. In addition, ComEst can estimate the completeness as a function of position on the input image at a given magnitude. i.e.,

$$
\mathrm{f}_{\text {com }}(x, y, m)=\frac{N_{\text {se }}(x, y, m)}{N_{\text {true }}(x, y, m)},
$$

where $\mathrm{f}_{\mathrm{com}}(x, y, m), N_{\mathrm{se}}(x, y, m)$ and $N_{\text {true }}(x, y, m)$ are the completeness, number of simulated sources re-covered by SExtractor and the number of the input sources in the simulation, respectively, at the magnitude of $m$ and the coordinate $(x, y)$ of the input image. Precisely, we derive the spatial distribution of number counts (e.g., $N_{\mathrm{se}}\left(x_{i}, y_{j}, m_{k}\right)$ with the running indices $\left.i, j, k\right)$ by binning the boxes $\left(\left|x-x_{i}\right|<\Delta x / 2\right.$ and $\left.\left|y-y_{j}\right|<\Delta y / 2\right)$ in the Cartesian coordinates based on the sources in the magnitude range of $\left|m-m_{k}\right|<\Delta m / 2, \Delta x, \Delta y$ and $\Delta m$ are respectively the bin width of $x$-, $y$-coordinates and magnitude set up by the users.

On the other hand, ComEst can estimate the purity of the source detection in a similar manner. By comparing the catalogs produced by running SExtractor on the simulated images and the true catalogs used as inputs to the simulation, the purity of the source detection is derived as the fraction of sources detected by SExtractor which are also present in the input catalog of the simulation. Specifically, the purity of the source detection as a function of magnitude $\mathrm{f}_{\text {pur }}(m)$ and a function of position on the image give a magnitude cut $\mathrm{f}_{\text {pur }}(x, y, m)$ is defined as follows. i.e.,

$$
\begin{aligned}
\mathrm{f}_{\text {pur }}(m) & =\frac{N_{\text {se }}(m)}{N_{\text {se }}(m)+N_{\text {false }}(m)} \\
\mathrm{f}_{\text {pur }}(x, y, m) & =\frac{N_{\text {se }}(x, y, m)}{N_{\text {se }}(x, y, m)+N_{\text {false }}(x, y, m)},
\end{aligned}
$$

where $N_{\text {false }}$ is the number of the false detections- the sources detected by SExtractor on the SFI which are not present in the true catalogs.

By defining the detection rate $f_{d r}$ as the ratio of the numbers of all detected sources to the input sources, the above equations can be simply connected as Equation 5. That is, the detection rate contaminated by the false detection (Type I error or false positive) is quantified as the completeness of the source detection.

$$
\begin{aligned}
\mathrm{f}_{\mathrm{dr}} & \equiv \frac{N_{\text {se }}+N_{\text {false }}}{N_{\text {true }}} \\
\mathrm{f}_{\text {com }} & \equiv \mathrm{f}_{\text {pur }} \times \mathrm{f}_{\text {dr }} .
\end{aligned}
$$

For calculating the completeness and purity, users can use a masked map to account for the masked area (e.g., by bright and saturated stars) which is present in the input image. If the pixel is masked, then it would not be included in the calculation. Note that the completeness and purity estimated by ComEst depend on the configurations which are used for simulating the point sources and galaxies. It is therefore important to estimate the completeness by requiring realistic configurations (e.g., the half-light radius or FWHM) in the simulation.

Since ComEst essentially re-detects the simulated sources and measures the photometry from end to end, in principle ComEst can also be used to test the photometry performance (e.g., the ZP calibration) of the observed image by investigating the simulated sources detected by ComEst. However, calibrating the photometry measurement is beyond the scope of this paper and we do not delve into it further. 


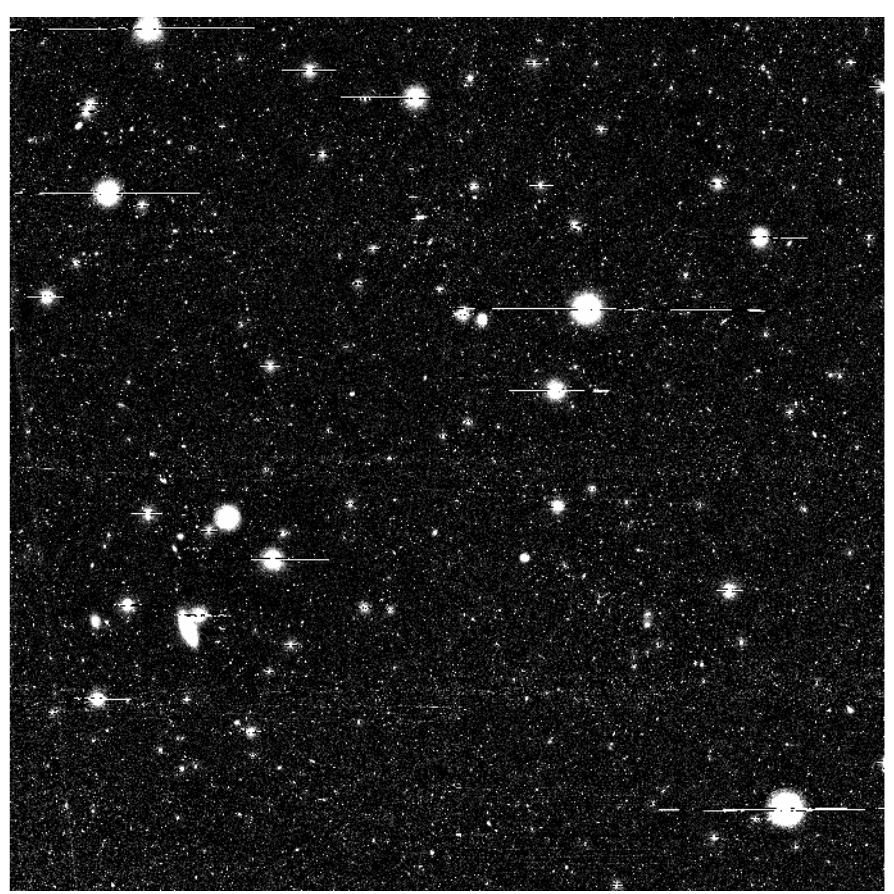

Figure 2: The image used as the example in this work. This image is the $i$-band imaging of the footprint $2329+0012$ observed by the Blanco Cosmology Survey, and the field of view is $\approx 35.5 \times 35.5 \mathrm{arcmin}^{2}$ (corresponding to $0.35 \mathrm{deg}^{2}$ ) with the pixel scale of 0.26 acrsec/pixel. Note that the edge effects around the bright stars and the signatures of the chip gaps appear on Figure 5 . especially for the completeness maps.

\section{Example and Results}

We demonstrate the usage of ComEst in Section 3.1 and present the results in Section 3.2

\subsection{Example}

The usage of ComEst is described in detail in this section. Specifically, we demonstrate the usage of ComEst by running ComEst on the footprint of BCS2329 + 0012 observed by the Blanco Cosmology Survey (Desai et al. 2012) as an example. We show the example image in Figure 2. We assume that the observed image is called example_img.fits in the current directory (CWD). Moreover, all the output files produced by ComEst will be saved in the directory called outdir_example.

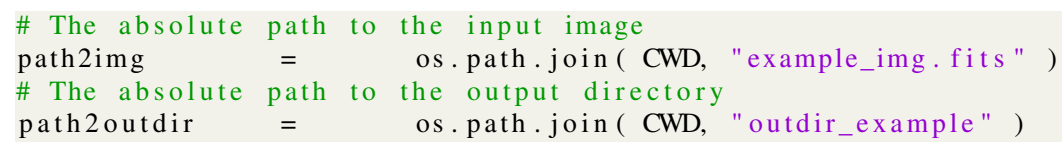

It is also required by ComEst to set the ZP of photometry img_zp (in units of magnitude), the pixel scale img_pixel_scale (in units of arcsec/pixel) and the FWHM of the observed image img_f whm (in the unit of arcsec).

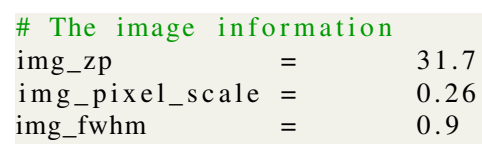

After the basic configuration is set (see above), we import the ComEst package and then load the input image with the required information.

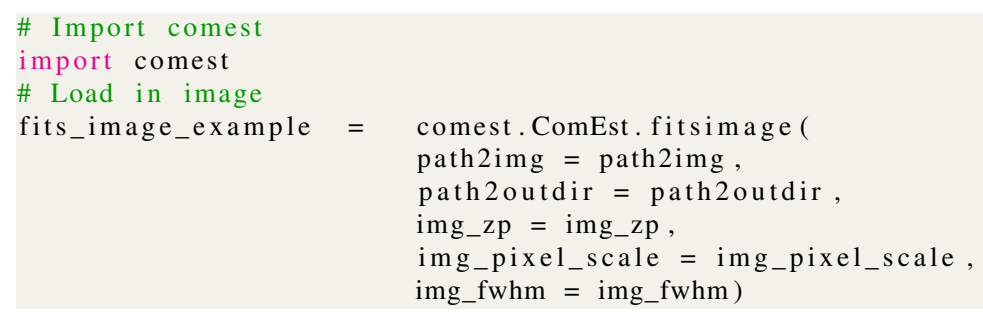

Once the image is loaded, we first run SExtractor on the input image to detect all the observed sources and then create the output catalog and check-images. The output files of the first SExtractor run are associated with the branch name of full (i.e., a set of output files with the file names containing full). After the first run of SExtractor, we can create the SFI (see Section 2.11 with the file name of full.identical.srcfree.fits. Optionally, ComEst can create the "BnB" image which only contains the observed "Bright and Big" sources, with their SNR and size larger than the assigned threshold (see Section 2.11. All the output files at this stage are saved in the directory of outdir_example. 


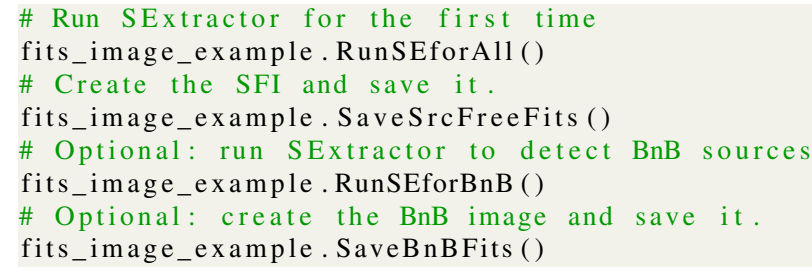

Next, we simulate the sources on the SFI image using the simulation toolkit GalSim (see Section 2.2). For the purpose of illustration, we only demonstrate the case of simulating the galaxies which consist of bulge and disk components. The same configuration scheme can be applied for simulating point sources and re-sampling the galaxies from the COSMOS catalog. The simulated galaxies are uniformly sampled in the user-defined interval of magnitude, half-light radius, fraction of bulge component, minor-to-major axis ratio, and the position angle on the sky. In this case we use the default values of ComEst that the half-light radius and minor-to-major axis ratio span a range of $\left(0.35^{\prime \prime}, 0.75^{\prime \prime}\right)$ and $(0.4,1.0)$, respectively. ComEst can simulate multiple images with the same configuration scheme in parallel by setting the arguments nsimimages and ncpu. For example, one can simulate 10 images using $5 \mathrm{CPU}$ cores by setting nsimimages $=10$ and ncpu $=5$. The number density of the simulated sources per arcmin square (ngals_arcmin2) can be provided by the users. It is worth mentioning that one should pay attention to the number density of the simulated sources because the completeness of the source detection will be inevitably affected by blending in the crowded field simulated by ComEst. In this example, we set the number density of the simulated sources to be 15 per arcmin square (ngals_arcmin2 = 15.0). In this simulation, all the output files (simulated images and true catalogs) are associated with the branch name of buldisk (by setting sims_nameroot = "buldisk").

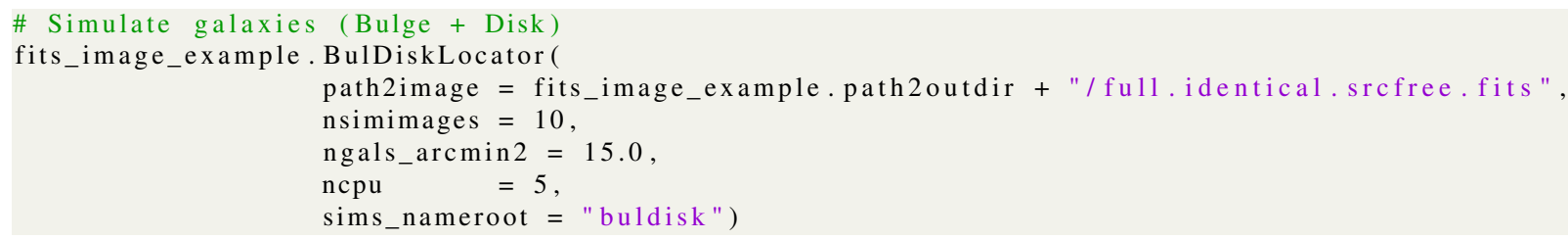

After the simulation tasks, we re-run SExtractor on the 10 simulated images associated with the branch name of buldisk and output the resulting catalogs merged from the 10 SExtractor runs. This is done by the following command.

\# Run SExtractor on the simulated images with the branch name of "buldisk"

fits_image_example.RunSEforSims (sims_nameroot = "buldisk")

Four catalogs associated to the branch name (i.e., buldisk for this case) are outputted, as listed in Table 1

In the end, using the output catalogs listed in Table 1 we derive the completeness of the source detection as a function of magnitude (Equation 1) and CCD position (Equation 2) by invoking the following command.

\# Derive the completeness of the source detection for images of the branch name "buldisk" fits_image_example.DeriveCom $\left(\operatorname{sims} \_n a m e r o o t=" b u l d i s k "\right)$

The completeness of the source detection is derived as the ratio of the source counts re-detected by ComEst (*.matched_pairs.cat.fits) to the source counts in the input catalog of the simulation (*.merged_true.cat.fits) for a given magnitude cut and CCD position. On the other hand, ComEst can also derive the purity of the source detection- which is defined by the ratio of the simulated source counts re-detected by SExtractor (*.sims.sex.matched_pairs.cat.fits) to the source counts of all detections (*.sims.sex.matched_pairs.cat.fits +*.sims.sex.ghost.cat.fits). This can be done by invoking

\# Derive the purity of the source detection for images of the branch name "buldisk"

fits_image_example.DerivePur(sims_nameroot = "buldisk")

We present the results in the following section.

Table 1: The output catalogs of running SExtractor on the simulated images associated with the branch name buldisk by executing the method of RunSEf orSims.

\begin{tabular}{ll}
\hline Name & Definition \\
\hline \hline buldisk.sims.sex.merged_true.cat.fits & The merged true catalog of the simulated sources. \\
buldisk.sims.sex.matched_pairs.cat.fits & The merged catalog of the simulated sources re-detected by SExtractor. \\
buldisk.sims.sex.unmatched.cat.fits & The merged catalog of the simulated sources which are not re-detected by SExtractor. \\
buldisk.sims.sex.ghost.cat.fits & The merged catalog of the sources which are detected by SExtractor \\
& but are not in the true catalog (i.e., the false detection)
\end{tabular}

\subsection{Results}

The simulated images and the performance of photometry estimated by SExtractor are presented in Section 3.2.1. We show the results in Section 3.2 .2 

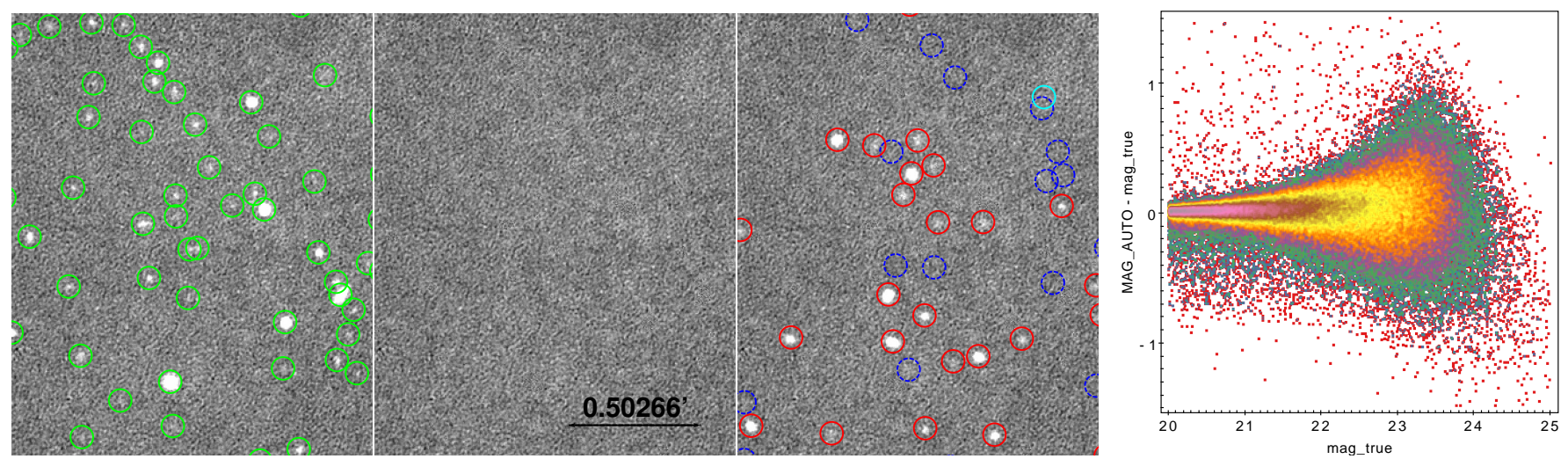

Figure 3: The observed/simulated images and the derived photometry. First panel: the observed image with the detected sources marked by green circles. Second panel: the derived SFI (see Section 2.1. No clear source is seen above the sky noise level. Third panel: the simulated image by placing simulated galaxies (bulge + disk) on the derived SFI. The red circles show the simulated sources which are re-detected by SExtractor, while the blue-dashed circles indicate the non-detected sources simulated on the image. The false detection (i.e., the sources are detected by SExtractor on the simulated image but are not in the input catalog of the simulation) is marked by the cyan circle. The scatter plot on the right is the MAG_AUTO photometry estimated by SExtractor compared to the input magnitude of the simulated sources. The $y$-axis is the MAG_AUTO photometry estimated by SExtractor, while the $x$-axis is the input magnitude mag_true in the GalSim simulation. The density of the detected sources in the MAG_AUTO v.s. mag_true plane is plotted. The black solid line shows the equality line.

\subsubsection{Diagnostic outputs}

The observed/simulated images and the derived photometry are shown in Figure 3 . In this simulation we simulate galaxies with magnitudes spanning a range between 20 mag and 27 mag. The cutout of the observed tile BCS2329+0012 (the first panel), derived SFI (the second panel) and one out of 10 simulated images (the third panel) are shown in Figure 3 As seen in Figure 3 , the observed sources (green circles) are clearly detected by SExtractor in the observed image and are then replaced by the estimated background noise to produce SFI, as shown in the second panel. No clear signal above the observed sky noise is seen in the SFI image. The galaxies consisting of the bulge and disk components are simulated on the derived SFI, as shown by the cutout image in the third panel. The simulated sources detected by SExtractor are marked by the red circles, while the bluedashed circles indicate the sources, which are simulated but are not detected. The false detection is marked by the cyan circle in the third panel. In the fourth panel of Figure 3 we show the comparison between the photometry estimated by SExtractor and the input magnitude of the simulated sources. Specifically, we compare the MAG_AUTO photometry to the input magnitude mag_true. The result suggests that the photometry performance is unbiased. In addition, the clear drop of the source density in the plane of MAG_AUTO v.s. mag_true is seen, indicating that the source detection starts to suffer from the incompleteness at mag_true $\approx 23.5$ mag.

\subsubsection{Completeness and Purity}

We first show the completeness $\mathrm{f}_{\text {com }}(m)$ and purity $\mathrm{f}_{\text {pur }}(m)$ of the source detection as a function of magnitude in Figure 4 . In the left panel of Figure 4, we also show the completeness of point sources and galaxies re-sampled from the observed COSMOS catalog (see Section 2.2p estimated by ComEst. In addition, the limiting magnitudes calculated using the $10 \sigma$ depths for point source and galaxies are taken from Desai et al.(2012) and the completeness of the source detection derived from the histogram counts (see Section 1) are also plotted for comparison. The completeness derived by ComEst agrees reasonably well for the case of galaxies, with the tendency that the magnitudes associated with the completeness levels derived by re-sampling the COSMOS catalog are slightly deeper (by $\approx 0.1-0.15 \mathrm{mag}$ ) than the results using the galaxies consisting of bulge and disk components and using the histogram count technique. The $10 \sigma$ depth of galaxies indicates the completeness at the level of $\approx 92 \%$ and $\approx 85 \%$ based on the estimates of ComEst and histogram count technique, respectively. On the other hand, the magnitudes which correspond to the same completeness level for the point sources are overall fainter than the galaxies by $\approx 1$ mag, which is consistent with the difference of the $10 \sigma$ depth seen between the galaxies and point sources. The purity of the source detection for point sources, galaxies consisting of the bulge and disk components and the galaxies re-sampled from COSMOS catalog are shown in the right panel of Figure 4 . There is no significant difference of the purity seen among the simulated samples, indicating that the $50 \%$ completeness corresponds to the purity of $\approx 94 \%$ and $\approx 72 \%$ for galaxies and point sources, respectively.

We show the results of completeness and purity of the source detection estimated by ComEst as functions of CCD position given the magnitude threshold in Figure 5. The completeness and purity maps are constructed by binning 1 arcmin in both $x$ and $y$ direction and are showed in Figure 5 after the bicubic interpolation. To get better statistics on a single cell basis in the magnitude and position space $(x, y, m)$, we simulate 100 images based on the derived SFI (see Section 2.2) in deriving the $\mathrm{f}_{\text {com }}(x, y, m)$ and $\mathrm{f}_{\text {pur }}(x, y, m)$. Note that the finner binning needs more simulated images to achieve enough statistics (i.e., the galaxy counts) in a single binned cell, hence the users should compromise between the desired resolution in the spatial variance and the running time spent on the image simulations. For simplicity, we only show the results of galaxies consisting of bulge and disk components (the blue solid lines in Figure 4), since the same qualitative picture is suggested by other cases estimated by ComEst. Specifically, we show the results of completeness (purity) as a function of CCD position given the magnitude cut in the intervals centering on $m=23 \mathrm{mag}, m=23.5 \mathrm{mag}$ and $m=24 \mathrm{mag}(m=23.5 \mathrm{mag}, m=24 \mathrm{mag}$ and $m=24.5 \mathrm{mag})$ with the width of $0.1 \mathrm{mag}$ in the upper (lower) panel of Figure 5 It is worth mentioning in Figure 5 that the edge effect around the bright stars seen in Figure 2 is captured by ComEst, while the chip gaps- which are not easy to be visually inspected in Figure 2 are clearly characterized in the completeness map. These signatures are less prominent in the purity map at the very faint end 

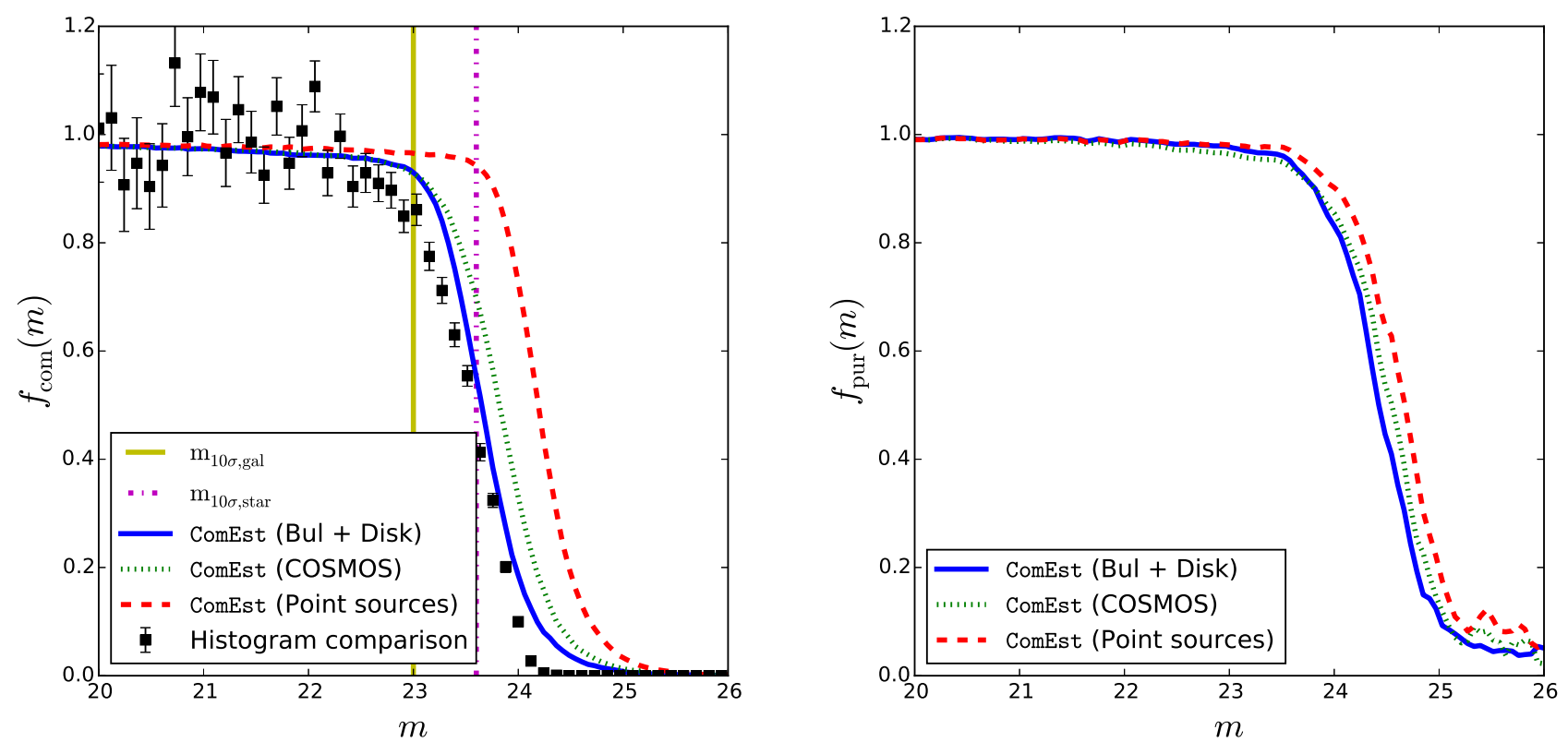

Figure 4: The completeness and purity of the source detection as functions of magnitude. Left panel: the completeness estimated by ComEst as a function of magnitude for the simulated galaxies consisting of bulge and disk components (blue solid line), galaxies re-sampled from the COSMOS catalog (green dotted line) and the simulated point sources (red dashed line). The completeness derived from the histogram counts is shown by black squares. The $10 \sigma$ depth of galaxies and point sources are indicated by the yellow solid line and magenta dot-dashed line, respectively. Right panel: the purity estimated by ComEst as functions of magnitude for the simulated galaxies consisting of bulge and disk components, galaxies re-sampled from the COSMOS catalog and the simulated point sources. The color codes are the same as the left panel are used.

(e.g., $\gtrsim 24.5 \mathrm{mag}$ ) because of the fact that the detection rate (i.e., Equation 5 is too low $\left(N_{\mathrm{se}}+N_{\mathrm{false}} \approx 0\right)$ to have the meaningful estimates of the purity, causing the purity maps consisting of the cells with $1 \times 1 \operatorname{arcmin}^{2}$ suffer from large poisson fluctuation. The resulting completeness and purity maps of the sourced detection show reasonable uniformity in the context of the spatial properties of the tile BCS2329+0012.

\section{Conclusions}

We have presented a python package ComEst to estimate the completeness and purity of the source extractor SExtractor used for the optical/NIR imaging of point sources or galaxies, by utilizing the state-of-the-art simulation toolkit GalSim. The observed image saved in the FITS format with a well-calibrated ZP is the only input required by ComEst. ComEst carries out the simulation and source detection directly on the observed image, which ensures that the information about the observational artifacts and noise properties are preserved while deriving the completeness and purity estimates. ComEst can simulate galaxies with various properties, such as half-light radius or minor-to-major axis ratio. The running time of the simulations conducted by ComEst is generally fast, it takes about $\approx 60$ seconds for simulating 15 galaxies/arcmin ${ }^{2}$ on a $0.35 \mathrm{deg}^{2}$ footprint with the pixel scale of $0.26 \mathrm{arcsec} / \mathrm{pixel}$. In addition, running multiple simulations in parallel is supported in ComEst. It is worth noting that ComEst estimates the completeness and purity of the source detection as a function of magnitude and CCD position in a fully automated fashion with an easy-to-use syntax. We expect that ComEst would be extremely helpful in quantifying the systematics related to the completeness and purity for the analysis such as the galaxy clustering or the lensing magnification.

ComEst is publicly available and can be downloaded from https://github.com/inonchiu/ComEst, along with full documentation and more detailed examples.

\section{Acknowledgements}

This paper is dedicated to Chien-Ho Lin in Taiwan. We acknowledge the support by the DFG Cluster of Excellence "Origin and Structure of the Universe", the DLR award 50 OR 1205 that supported I. Chiu during his PhD project, and the Transregio program TR33 "The Dark Universe". The computations for ComEst have been carried out on the computing facilities of the Computational Center for Particle and Astrophysics (C2PAP) and of the Leibniz Supercomputer Center (LRZ). 

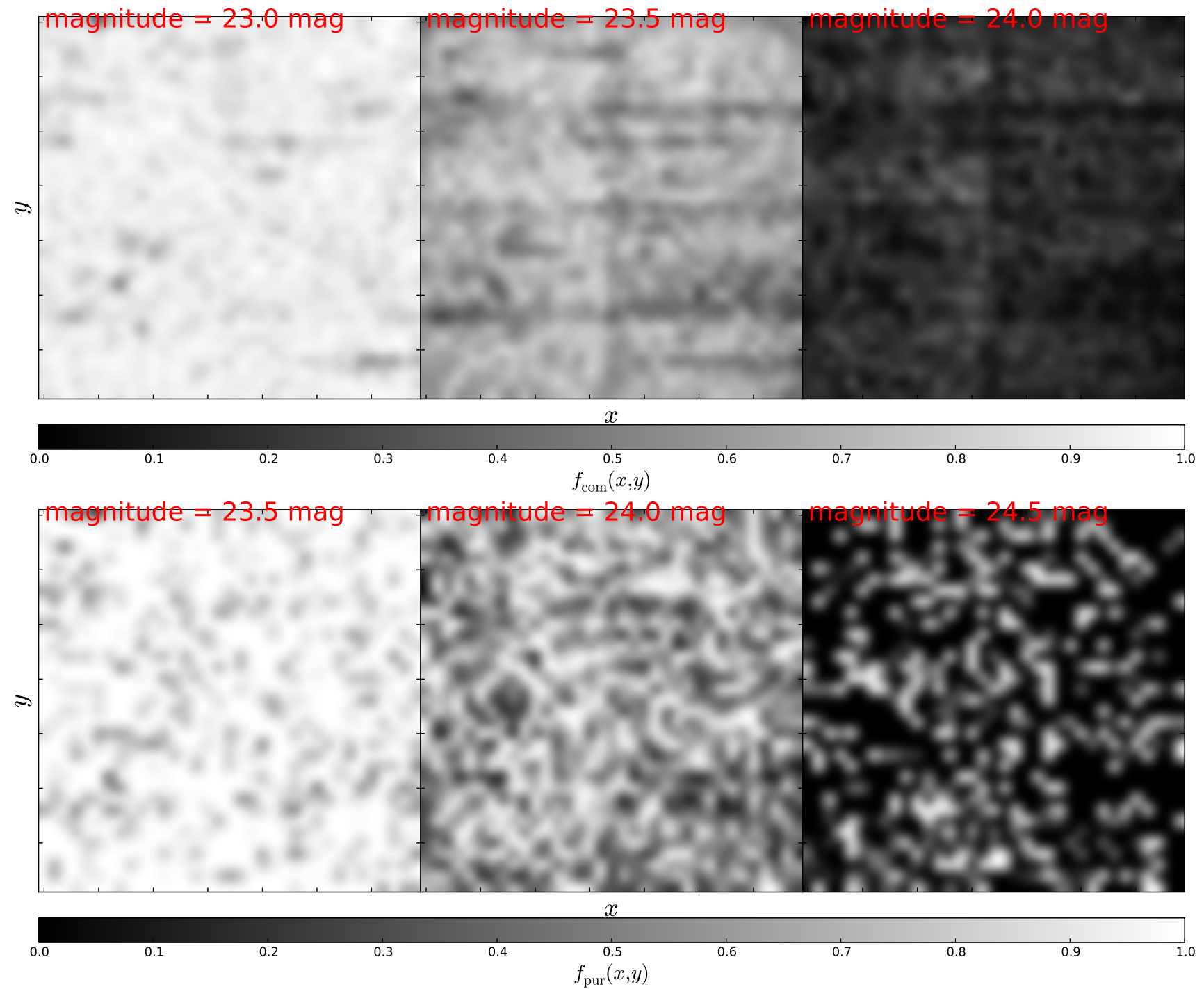

Figure 5: The completeness and purity maps estimated by ComEst with the footprint size of $35.5^{\prime} \times 35.5^{\prime}$. Left panel: the completeness map $(x, y)$ in the magnitude intervals centered on $m=23 \mathrm{mag}$ (left), $m=23.5 \mathrm{mag}$ (middle) and $m=24 \mathrm{mag}$ (right) with magnitude width $\Delta m=0.1 \mathrm{mag}$. The completeness of source detection is color-coded by the colorbar shown below the figures. Right panel: the purity map $(x, y)$ centered on $m=23.5 \mathrm{mag}$ (left), $m=24 \mathrm{mag}$ (middle) and $m=24.5 \mathrm{mag}$ (right). The colorbar is the same as completeness map is applied. 


\section{References}

\section{References}

D. G. York, J. Adelman, J. E. Anderson, Jr., S. F. Anderson, J. Annis, N. A. Bahcall, J. A. Bakken, R. Barkhouser, S. Bastian, E. Berman, W. N. Boroski, S. Bracker, C. Briegel, J. W. Briggs, J. Brinkmann, R. Brunner, S. Burles, L. Carey, M. A. Carr, F. J. Castander, B. Chen, P. L. Colestock, A. J. Connolly, J. H. Crocker, I. Csabai, P. C. Czarapata, J. E. Davis, M. Doi, T. Dombeck, D. Eisenstein, N. Ellman, B. R. Elms, M. L. Evans, X. Fan, G. R. Federwitz, L. Fiscelli, S. Friedman, J. A. Frieman, M. Fukugita, B. Gillespie, J. E. Gunn, V. K. Gurbani, E. de Haas, M. Haldeman, F. H. Harris, J. Hayes, T. M. Heckman, G. S. Hennessy, R. B. Hindsley, S. Holm, D. J. Holmgren, C.-h. Huang, C. Hull, D. Husby, S.-I. Ichikawa, T. Ichikawa, Ž. Ivezić, S. Kent, R. S. J. Kim, E. Kinney, M. Klaene, A. N. Kleinman, S. Kleinman, G. R. Knapp, J. Korienek, R. G. Kron, P. Z. Kunszt, D. Q. Lamb, B. Lee, R. F. Leger, S. Limmongkol, C. Lindenmeyer, D. C. Long, C. Loomis, J. Loveday, R. Lucinio, R. H. Lupton, B. MacKinnon, E. J. Mannery, P. M. Mantsch, B. Margon, P. McGehee, T. A. McKay, A. Meiksin, A. Merelli, D. G. Monet, J. A. Munn, V. K. Narayanan, T. Nash, E. Neilsen, R. Neswold, H. J. Newberg, R. C. Nichol, T. Nicinski, M. Nonino, N. Okada, S. Okamura, J. P. Ostriker, R. Owen, A. G. Pauls, J. Peoples, R. L. Peterson, D. Petravick, J. R. Pier, A. Pope, R. Pordes, A. Prosapio, R. Rechenmacher, T. R. Quinn, G. T. Richards, M. W. Richmond, C. H. Rivetta, C. M. Rockosi, K. Ruthmansdorfer, D. Sandford, D. J. Schlegel, D. P. Schneider, M. Sekiguchi, G. Sergey, K. Shimasaku, W. A. Siegmund, S. Smee, J. A. Smith, S. Snedden, R. Stone, C. Stoughton, M. A. Strauss, C. Stubbs, M. SubbaRao, A. S. Szalay, I. Szapudi, G. P. Szokoly, A. R. Thakar, C. Tremonti, D. L. Tucker, A. Uomoto, D. Vanden Berk, M. S. Vogeley, P. Waddell, S.-i. Wang, M. Watanabe, D. H. Weinberg, B. Yanny, N. Yasuda, and SDSS Collaboration, "The Sloan Digital Sky Survey: Technical Summary," AJ, vol. 120, pp. 1579-1587, Sep. 2000.

J. S. Morgan, W. Burgett, and P. Onaka, "The Pan-STARRS Project in 2014," in Society of Photo-Optical Instrumentation Engineers (SPIE) Conference Series, ser. Society of Photo-Optical Instrumentation Engineers (SPIE) Conference Series, vol. 9145, Jul. 2014.

B. Flaugher, “The Dark Energy Survey,” International Journal of Modern Physics A, vol. 20, pp. 3121-3123, 2005.

J. T. A. de Jong, G. A. Verdoes Kleijn, K. H. Kuijken, and E. A. Valentijn, “The Kilo-Degree Survey," Experimental Astronomy, vol. 35, pp. 25-44, Jan. 2013.

S. Miyazaki, Y. Komiyama, H. Nakaya, Y. Kamata, Y. Doi, T. Hamana, H. Karoji, H. Furusawa, S. Kawanomoto, T. Morokuma, Y. Ishizuka, K. Nariai, Y. Tanaka, F. Uraguchi, Y. Utsumi, Y. Obuchi, Y. Okura, M. Oguri, T. Takata, D. Tomono, T. Kurakami, K. Namikawa, T. Usuda, H. Yamanoi, T. Terai, H. Uekiyo, Y. Yamada, M. Koike, H. Aihara, Y. Fujimori, S. Mineo, H. Miyatake, N. Yasuda, J. Nishizawa, T. Saito, M. Tanaka, T. Uchida, N. Katayama, S.-Y. Wang, H.-Y. Chen, R. Lupton, C. Loomis, S. Bickerton, P. Price, J. Gunn, H. Suzuki, Y. Miyazaki, M. Muramatsu, K. Yamamoto, M. Endo, Y. Ezaki, N. Itoh, Y. Miwa, H. Yokota, T. Matsuda, R. Ebinuma, and K. Takeshi, "Hyper Suprime-Cam," in Society of Photo-Optical Instrumentation Engineers (SPIE) Conference Series, ser. Society of Photo-Optical Instrumentation Engineers (SPIE) Conference Series, vol. 8446, Sep. 2012.

M. F. Skrutskie, R. M. Cutri, R. Stiening, M. D. Weinberg, S. Schneider, J. M. Carpenter, C. Beichman, R. Capps, T. Chester, J. Elias, J. Huchra, J. Liebert, C. Lonsdale, D. G. Monet, S. Price, P. Seitzer, T. Jarrett, J. D. Kirkpatrick, J. E. Gizis, E. Howard, T. Evans, J. Fowler, L. Fullmer, R. Hurt, R. Light, E. L. Kopan, K. A. Marsh, H. L. McCallon, R. Tam, S. Van Dyk, and S. Wheelock, "The Two Micron All Sky Survey (2MASS)," AJ, vol. 131, pp. 1163-1183, Feb. 2006.

E. L. Wright, P. R. M. Eisenhardt, A. K. Mainzer, M. E. Ressler, R. M. Cutri, T. Jarrett, J. D. Kirkpatrick, D. Padgett, R. S. McMillan, M. Skrutskie, S. A. Stanford, M. Cohen, R. G. Walker, J. C. Mather, D. Leisawitz, T. N. Gautier, III, I. McLean, D. Benford, C. J. Lonsdale, A. Blain, B. Mendez, W. R. Irace, V. Duval, F. Liu, D. Royer, I. Heinrichsen, J. Howard, M. Shannon, M. Kendall, A. L. Walsh, M. Larsen, J. G. Cardon, S. Schick, M. Schwalm, M. Abid, B. Fabinsky, L. Naes, and C.-W. Tsai, “The Wide-field Infrared Survey Explorer (WISE): Mission Description and Initial On-orbit Performance," AJ, vol. 140, pp. 1868-1881, Dec. 2010.

T. Shanks, N. Metcalfe, B. Chehade, J. R. Findlay, M. J. Irwin, E. Gonzalez-Solares, J. R. Lewis, A. K. Yoldas, R. G. Mann, M. A. Read, E. T. W. Sutorius, and S. Voutsinas, “The VLT Survey Telescope ATLAS,” MNRAS, vol. 451, pp. 4238-4252, Aug. 2015.

A. M. Koekemoer, H. Aussel, D. Calzetti, P. Capak, M. Giavalisco, J.-P. Kneib, A. Leauthaud, O. Le Fèvre, H. J. McCracken, R. Massey, B. Mobasher, J. Rhodes, N. Scoville, and P. L. Shopbell, "The COSMOS Survey: Hubble Space Telescope Advanced Camera for Surveys Observations and Data Processing,” ApJS, vol. 172, pp. 196-202, Sep. 2007.

S. V. W. Beckwith, M. Stiavelli, A. M. Koekemoer, J. A. R. Caldwell, H. C. Ferguson, R. Hook, R. A. Lucas, L. E. Bergeron, M. Corbin, S. Jogee, N. Panagia, M. Robberto, P. Royle, R. S. Somerville, and M. Sosey, “The Hubble Ultra Deep Field,” AJ, vol. 132, pp. 1729-1755, Nov. 2006.

Z. Ivezic, J. A. Tyson, B. Abel, E. Acosta, R. Allsman, Y. AlSayyad, S. F. Anderson, J. Andrew, R. Angel, G. Angeli, R. Ansari, P. Antilogus, K. T. Arndt, P. Astier, E. Aubourg, T. Axelrod, D. J. Bard, J. D. Barr, A. Barrau, J. G. Bartlett, B. J. Bauman, S. Beaumont, A. C. Becker, J. Becla, C. Beldica, S. Bellavia, G. Blanc, R. D. Blandford, J. S. Bloom, J. Bogart, K. Borne, 
J. F. Bosch, D. Boutigny, W. N. Brandt, M. E. Brown, J. S. Bullock, P. Burchat, D. L. Burke, G. Cagnoli, D. Calabrese, S. Chandrasekharan, S. Chesley, E. C. Cheu, J. Chiang, C. F. Claver, A. J. Connolly, K. H. Cook, A. Cooray, K. R. Covey, C. Cribbs, W. Cui, R. Cutri, G. Daubard, G. Daues, F. Delgado, S. Digel, P. Doherty, R. Dubois, G. P. Dubois-Felsmann, J. Durech, M. Eracleous, H. Ferguson, J. Frank, M. Freemon, E. Gangler, E. Gawiser, J. C. Geary, P. Gee, M. Geha, R. R. Gibson, D. K. Gilmore, T. Glanzman, I. Goodenow, W. J. Gressler, P. Gris, A. Guyonnet, P. A. Hascall, J. Haupt, F. Hernandez, C. Hogan, D. Huang, M. E. Huffer, W. R. Innes, S. H. Jacoby, B. Jain, J. Jee, J. G. Jernigan, D. Jevremovic, K. Johns, R. L. Jones, C. Juramy-Gilles, M. Juric, S. M. Kahn, J. S. Kalirai, N. Kallivayalil, B. Kalmbach, J. P. Kantor, M. M. Kasliwal, R. Kessler, D. Kirkby, L. Knox, I. Kotov, V. L. Krabbendam, S. Krughoff, P. Kubanek, J. Kuczewski, S. Kulkarni, R. Lambert, L. Le Guillou, D. Levine, M. Liang, K. Lim, C. Lintott, R. H. Lupton, A. Mahabal, P. Marshall, S. Marshall, M. May, R. McKercher, M. Migliore, M. Miller, D. J. Mills, D. G. Monet, M. Moniez, D. R. Neill, J. Nief, A. Nomerotski, M. Nordby, P. O’Connor, J. Oliver, S. S. Olivier, K. Olsen, S. Ortiz, R. E. Owen, R. Pain, J. R. Peterson, C. E. Petry, F. Pierfederici, S. Pietrowicz, R. Pike, P. A. Pinto, R. Plante, S. Plate, P. A. Price, M. Prouza, V. Radeka, J. Rajagopal, A. Rasmussen, N. Regnault, S. T. Ridgway, S. Ritz, W. Rosing, C. Roucelle, M. R. Rumore, S. Russo, A. Saha, B. Sassolas, T. L. Schalk, R. H. Schindler, D. P. Schneider, G. Schumacher, J. Sebag, G. H. Sembroski, L. G. Seppala, I. Shipsey, N. Silvestri, J. A. Smith, R. C. Smith, M. A. Strauss, C. W. Stubbs, D. Sweeney, A. Szalay, P. Takacs, J. J. Thaler, R. Van Berg, D. Vanden Berk, K. Vetter, F. Virieux, B. Xin, L. Walkowicz, C. W. Walter, D. L. Wang, M. Warner, B. Willman, D. Wittman, S. C. Wolff, W. M. Wood-Vasey, P. Yoachim, H. Zhan, and for the LSST Collaboration, "LSST: from Science Drivers to Reference Design and Anticipated Data Products," ArXiv e-prints, May 2008.

R. Laureijs, J. Amiaux, S. Arduini, J. . Auguères, J. Brinchmann, R. Cole, M. Cropper, C. Dabin, L. Duvet, A. Ealet, and et al., "Euclid Definition Study Report," ArXiv e-prints, Oct. 2011.

E. Bertin and S. Arnouts, “SExtractor: Software for source extraction.” AAPS, vol. 117, pp. 393-404, Jun. 1996.

A. Zenteno, J. Song, S. Desai, R. Armstrong, J. J. Mohr, C.-C. Ngeow, W. A. Barkhouse, S. S. Allam, K. Andersson, G. Bazin, B. A. Benson, E. Bertin, M. Brodwin, E. J. Buckley-Geer, S. M. Hansen, F. W. High, H. Lin, Y.-T. Lin, J. Liu, A. Rest, R. C. Smith, B. Stalder, A. A. Stark, D. L. Tucker, and Y. Yang, "A Multiband Study of the Galaxy Populations of the First Four Sunyaev-Zel'dovich Effect Selected Galaxy Clusters,” ApJ, vol. 734, p. 3, Jun. 2011.

I. Chiu, J. P. Dietrich, J. Mohr, D. E. Applegate, B. A. Benson, L. E. Bleem, M. B. Bayliss, S. Bocquet, J. E. Carlstrom, R. Capasso, S. Desai, C. Gangkofner, A. H. Gonzalez, N. Gupta, C. Hennig, H. Hoekstra, A. von der Linden, J. Liu, M. McDonald, C. L. Reichardt, A. Saro, T. Schrabback, V. Strazzullo, C. W. Stubbs, and A. Zenteno, "Detection of enhancement in number densities of background galaxies due to magnification by massive galaxy clusters," MNRAS, vol. 457, pp. 3050-3065, Apr. 2016.

E. S. Rykoff, E. Rozo, and R. Keisler, “Assessing Galaxy Limiting Magnitudes in Large Optical Surveys,” ArXiv e-prints, Sep. 2015.

P. B. Stetson, "DAOPHOT - A computer program for crowded-field stellar photometry,” PASP, vol. 99, pp. 191-222, Mar. 1987.

F. La Barbera, R. R. de Carvalho, J. L. Kohl-Moreira, R. R. Gal, M. Soares-Santos, M. Capaccioli, R. Santos, and N. Sant'anna, "2DPHOT: A Multi-Purpose Environment for the Two-Dimensional Analysis of Wide-Field Images," PASP, vol. 120, pp. 681-702, May 2008.

B. T. P. Rowe, M. Jarvis, R. Mandelbaum, G. M. Bernstein, J. Bosch, M. Simet, J. E. Meyers, T. Kacprzak, R. Nakajima, J. Zuntz, H. Miyatake, J. P. Dietrich, R. Armstrong, P. Melchior, and M. S. S. Gill, "GALSIM: The modular galaxy image simulation toolkit," Astronomy and Computing, vol. 10, pp. 121-150, Apr. 2015.

B. W. Holwerda, “Source Extractor for Dummies v5,” ArXiv Astrophysics e-prints, Dec. 2005.

K. Umetsu, T. Broadhurst, A. Zitrin, E. Medezinski, and L.-Y. Hsu, "Cluster Mass Profiles from a Bayesian Analysis of Weaklensing Distortion and Magnification Measurements: Applications to Subaru Data,” ApJ, vol. 729, p. 127, Mar. 2011.

O. Ilbert, P. Capak, M. Salvato, H. Aussel, H. J. McCracken, D. B. Sanders, N. Scoville, J. Kartaltepe, S. Arnouts, E. Le Floc'h, B. Mobasher, Y. Taniguchi, F. Lamareille, A. Leauthaud, S. Sasaki, D. Thompson, M. Zamojski, G. Zamorani, S. Bardelli, M. Bolzonella, A. Bongiorno, M. Brusa, K. I. Caputi, C. M. Carollo, T. Contini, R. Cook, G. Coppa, O. Cucciati, S. de la Torre, L. de Ravel, P. Franzetti, B. Garilli, G. Hasinger, A. Iovino, P. Kampczyk, J.-P. Kneib, C. Knobel, K. Kovac, J. F. Le Borgne, V. Le Brun, O. L. Fèvre, S. Lilly, D. Looper, C. Maier, V. Mainieri, Y. Mellier, M. Mignoli, T. Murayama, R. Pellò, Y. Peng, E. Pérez-Montero, A. Renzini, E. Ricciardelli, D. Schiminovich, M. Scodeggio, Y. Shioya, J. Silverman, J. Surace, M. Tanaka, L. Tasca, L. Tresse, D. Vergani, and E. Zucca, "Cosmos Photometric Redshifts with 30-Bands for 2-deg"," ApJ, vol. 690, pp. 1236-1249, Jan. 2009.

S. Desai, R. Armstrong, J. J. Mohr, D. R. Semler, J. Liu, E. Bertin, S. S. Allam, W. A. Barkhouse, G. Bazin, E. J. Buckley-Geer, M. C. Cooper, S. M. Hansen, F. W. High, H. Lin, Y.-T. Lin, C.-C. Ngeow, A. Rest, J. Song, D. Tucker, and A. Zenteno, “The Blanco Cosmology Survey: Data Acquisition, Processing, Calibration, Quality Diagnostics, and Data Release," ApJ, vol. 757, p. 83, Sep. 2012. 